\title{
Affine Connections, and Midpoint Formation
}

\author{
Anders Kock \\ Department of Mathematical Sciences, University of Aarhus \\ Ny Munkegade 118, Building 1530, DK-8000 Aarhus C \\ kock@imf .au.dk
}

\section{Preface}

It is a striking fact that differential calculus exists not only in analysis (based on the real numbers $\mathbb{R}$ ), but also in algebraic geometry, where no limit processes are available. In algebraic geometry, one rather uses the idea of nilpotent elements in the "affine line" $R$; they act as infinitesimals. (Recall that an element $x$ in a ring $R$ is called nilpotent if $x^{k}=0$ for suitable non-negative integer $k$.)

Synthetic differential geometry (SDG) is an axiomatic theory, based on such nilpotent infinitesimals. It can be proved, via topos theory, that the axiomatics covers both the differential-geometric notions of algebraic geometry and those of calculus.

I shall provide a glimpse of this synthetic method, by discussing its application to two particular types of differential-geometric structure, namely that of affine connection and of midpoint formation.

I shall not go much into the foundations of SDG, whose core is the so-called KI 11 axiom scheme. This is a very strong kind of axiomatics; in fact, a salient feature of it is: it is inconsistent - if you allow yourself the luxury of reasoning with so-called classical logic, i.e. use the "law of excluded middle", "proof by contradiction", etc. Rather, in SDG, one uses a weaker kind of logic, often called "constructive" or "intuitionist". Note the evident logical fact that there is a trade-off: with a weaker logic, stronger axiom systems become consistent. For the SDG axiomatics, it follows for instance that any function from the number line to itself is infinitely often differentiable (smooth); a very useful simplifying feature in differential geometry - but incompatible with the law of excluded middle, which allows you to construct the non-smooth function

$$
f(x)=\left\{\begin{array}{ll}
1 & \text { if } x=0, \\
0 & \text { if not }
\end{array} .\right.
$$

\section{Nilpotents, and Neighbours}

Nilpotent elements on the number line serve as infinitesimal 2 , in a sense which is "forbidden" when the number line is $\mathbb{R}$. Nilpotent infinitesimals come in a

\footnotetext{
${ }^{1}$ For "Kock-Lawvere".

2 They are not to be compared to the infinitesimals of non-standard analysis.
}

S. Brlek, C. Reutenauer, and X. Provençal (Eds.): DGCI 2009, LNCS 5810, pp. 13-21, 2009.

(C) Springer-Verlag Berlin Heidelberg 2009 
precise hierachy, since

$$
x^{k}=0 \quad \text { implies } \quad x^{k+1}=0 .
$$

The method of SDG combines the "nilpotency" ideas from algebraic geometry, with category theory, and categorical logic: category theory has provided a sense by which reasoning in (constructive) naive set theory is sound for geometric reasoning. So the following is formulated in such naive set theoretic terms.

We plunge directly into the geometry of infinitesimals (in the "nilpotency" sense): let us denote by $D \subseteq R$ the set of $x \in R$ with $x^{2}=0$ (the "first order infinitesimals"), more generally, let $D_{k} \subseteq R$ be the set of $k$ th order infinitesimals, meaning the set of $x \in R$ with $x^{k+1}=0$. (So $D=D_{1}$.) The basic instance of the KL axiom scheme says that any map $D_{k} \rightarrow R$ extends uniquely to a polynomial map $R \rightarrow R$ of degree $\leq k$. Thus, given any map $f: R \rightarrow R$, the restriction of $f$ to $D_{k}$ extends uniquely to a polynomial map of degree $\leq k$, the $k$ th Taylor polynomial of $f$ at 0 .

For $x$ and $y$ in $R$, we say that $x$ and $y$ are $k$ th order neighbours if $x-y \in D_{k}$, and we write $x \sim_{k} y$. It is clear that $\sim_{k}$ is a reflexive and symmetric relation. It is not transitive. For instance, if $x \in D$ and $y \in D$, then $x+y \in D_{2}$, by binomial expansion of $(x+y)^{3}$; but we cannot conclude $x+y \in D$. So $x \sim_{1} y$ and $y \sim_{1} z$ imply $x \sim_{2} z$, and similarly for higher $k$.

We now turn to the (first order) neighbour relations in the coordinate plane $R^{2}$. It is, in analogy with the 1-dimensional case, defined in terms of a subset $D(2) \subseteq R^{2}$; we put

$$
D(2)=\left\{\left(x_{1}, x_{2}\right) \in R \times R \mid x_{1}^{2}=0, x_{2}^{2}=0, x_{1} \cdot x_{2}=0\right\} ;
$$

we define $\underline{x} \sim \underline{y}$ if $\underline{x}-\underline{y} \in D(2)$, where $\underline{x}=\left(x_{1}, x_{2}\right)$ and $\underline{y}=\left(y_{1}, y_{2}\right)$. So $D(2) \subseteq D \times D$. Similarly for $D(n) \subseteq R^{n}$, and the resulting first order neighbour relation on the higher "coordinate vector spaces" $R^{n}$.

The following is a consequence of the $\mathrm{KL}$ axiom scheme:

Theorem 1.1. Any map $f: R^{n} \rightarrow R^{m}$ preserves the $k$ th order neighbour relation,

$$
\underline{x} \sim_{k} \underline{y} \quad \text { implies } \quad f(\underline{x}) \sim_{k} f(\underline{y}) .
$$

Proof sketch. for $n=2, m=1$, for the first order neighbour relation $\sim_{1}$. It suffices to see that $\underline{x} \sim_{1} \underline{0}$ implies $f(\underline{x}) \sim_{1} f(\underline{0})$, i.e to prove that $\underline{x} \in D(2)$ implies $f(\underline{x})-f(\underline{0}) \in D$. Now from a suitable version of the KL axiom scheme follows that on $D(2), f$ agrees with a unique affine function $T_{1} f: R^{2} \rightarrow R$, so

$$
f(\underline{x})-f(\underline{0})=a_{1} x_{1}+a_{2} x_{2} .
$$

Squaring the right hand side here yields 0 , since not only $x_{1} \in D$ and $x_{2} \in D$, but also $x_{1} \cdot x_{2}=0$. So $f(\underline{x})-f(\underline{0}) \in D$. 
From the Theorem follows that the relation $\sim_{k}$ on $R^{n}$ is coordinate free, i.e. is a truly geometric notion: any re-coordinatization of $R^{n}$ (by any map, not just by a linear or affine one) preserves the relation $\sim_{k}$.

For suitable definition of what an open subsets of $R^{n}$ is, and for a suitable definition of " $n$-dimensional manifold" (something that locally can be coordinatized with open subsets of $R^{n}$ ), one concludes that on any manifold, there are canonical reflexive symmetric relations $\sim_{k}$ : they may be defined in terms of a local coordinatization, but, by the Theorem, are independent of the coordinatization chosen.

Any map between manifolds preserves the relations $\sim_{k}$.

We shall mainly be interested in the first order neighbour relation $\sim_{1}$, which we shall also write just $\sim$. In Section 3 , we study aspects of the second order neighbour relation $\sim_{2}$.

So for a manifold $M$, we have a subset $M_{(1)} \subseteq M \times M$, the "first neighbourhood of the diagonal", consisting of $(x, y) \in M \times M$ with $x \sim y$. It was in terms of this "scheme" $M_{(1)}$ that algebraic geometers in the 1950 gave nilpotent infinitesimals a rigourous role in geometry. Note that for $M=R^{n}$, we have $M_{(1)} \cong M \times D(n)$, by the $\operatorname{map}(\underline{x}, \underline{y}) \mapsto(\underline{x}, \underline{x}-\underline{y})$.

Let us consider some notions from "infinitesimal geometry" which can be expressed in terms of the first order neighbour relation $\sim$ on an arbitrary manifold $M$. Given three points $x, y, z$ in $M$. If $x \sim y$ and $x \sim z$ we call the triple $(x, y, z)$ a 2-whisker at $x$ (sometimes: an infinitesimal 2-whisker, for emphasis); since $\sim$ is not transitive, we cannot in general conclude that $y \sim z$; if $y$ happens to be $\sim z$, we call the triple $(x, y, z)$ a 2-simplex (sometimes an infinitesimal 2-simplex). Similarly for $k$-whiskers and $k$-simplices. A $k$-simplex is thus a $k+1$-tuple of mutual neighbour points. The $k$-simplices form, as $k$ ranges, a simplicial complex, which in fact contains the information of differential forms, and the de Rham complex of $M$, see [2, 6], 1], 7].

(When we say that $\left(x_{0}, x_{1}, \ldots, x_{k}\right)$ is a $k$-whisker, we mean to say that it is a $k$-whisker at $x_{0}$, i.e. that $x_{0} \sim x_{i}$ for all $i=1, \ldots, k$. On the other hand, in a simplex, none of the points have a special status.)

Given a $k$-whisker $\left(x_{0}, \ldots, x_{k}\right)$ in $M$. If $U$ is an open subset of $M$ containing $x_{0}$, it will also contain the other $x_{i}$ s, and if $U$ is coordinatized by $R^{n}$, we may use coordinates to define the affine combination

$$
\sum_{i=0}^{k} t_{i} \cdot x_{i}
$$

(where $\sum t_{i}=1$; recall that this is the condition that a linear combination deserves the name of affine combination). The affine combination (1.1) can again be proved to belong to $U$, and thus it defines a point in $M$. The point thus obtained has in general not a good geometric significance, since it will depend on the coordinatization chosen. However (cf. [5], 7] 2.1), it does, if the whisker is a simplex:

Theorem 1.2. Let $\left(x_{0}, \ldots, x_{k}\right)$ be a $k$-simplex in $M$. Then the affine combination (1.1) is independent of the coordinatization used to define it. All the points 
that arise in this way are mutual neigbours. And any map to another manifold $M^{\prime}$ preserves such combinations.

Proof sketch. This is much in the spirit of the proof of Theorem 1.1 it suffices to see that any map $R^{n} \rightarrow R^{m}$ (not just a linear or affine one) preserves affine combinations of mutual neighbour points. This follows by considering a suitable first Taylor polynomial of $f$ (expand from $x_{0}$ ), and using the following purely algebraic fact: If $\underline{x}_{1}, \ldots, \underline{x}_{k}$ are in $D(n)$, then any linear combination of them will again be in $D(n)$ provided the $\underline{x}_{i}$ s are mutual neighbours.

Examples. If $x \sim y$ in a manifold (so they form a 1-simplex), we have the affine combinations "midpoint of $x$ and $y$ ", and "reflection of $x$ in $y$ ",

$$
\frac{1}{2} x+\frac{1}{2} y \quad \text { and } \quad 2 y-x,
$$

respectively. If $x, y, z$ form a 2 -simplex, we may form the affine combination $u:=y-x+z ;$ geometrically, it means completing the simplex into a parallelogram by adjoining the point $u$. Here is the relevant picture:

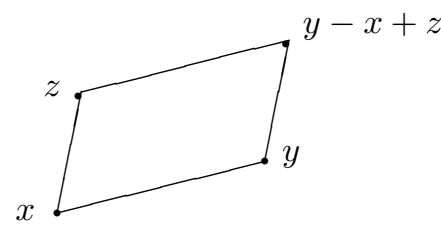

(All four points here are neighbours, not just those that are connected by lines in the figure.) The $u$ thus constructed will be a neighbour of each of the three given points. Therefore, we may form the midpoint of $x$ and $u$, and also we may form the midpoint of $y$ and $z$; these two midpoints will agree, because they do so in $R^{n}$, from where the construction of affine combinations was imported.

Remark. If $x, y, z$ and $u$ are as above, and if $x, y$, and $z$ belong to a subset $S \subseteq M$ given as a zero set of a function $f: M \rightarrow R$, then so does $u=y-x+z$; for, $f$ preserves this affine combination.

\section{Affine Connections}

If $x, y, z$ form a 2 -whisker at $x$ (so $x \sim y$ and $x \sim z$ ), we cannot canonically form a parallelogram as in (1.2); rather, parallelogram formation is an added structure:

Definition 2.1. An affine connection on a manifold $M$ is a law $\lambda$ which to any 2-whisker $x, y, z$ in $M$ associates a point $u=\lambda(x, y, z) \in M$, subject to the conditions

$$
\lambda(x, x, z)=z, \quad \lambda(x, y, x)=y .
$$


It can be verified, by working in a coordinatized situation, that several other laws follow; in a more abstract combinatorial situation than manifolds, these laws should probably be postulated. The laws are that for any 2 -whisker $(x, y, z)$

$$
\begin{gathered}
\lambda(x, y, z) \sim y \text { and } \lambda(x, y, z) \sim z \\
\lambda(y, x, \lambda(x, y, z))=z
\end{gathered}
$$

One will not in general have or require the "symmetry" condition

$$
\lambda(x, y, z)=\lambda(x, z, y)
$$

nor do we in general have, for 2 -simplices $x, y, z$, that

$$
\lambda(x, y, z)=y-x+z .
$$

The laws (2.4) and (2.5) are in fact equivalent, and affine connections satisfying either are called symmetric or torsion free. We return to the torsion of an affine connection below.

If $x, y, z, u$ are four points in $M$ such that $(x, y, z)$ is a 2-whisker at $x$, the statement $u=\lambda(x, y, z)$ can be rendered by a diagram

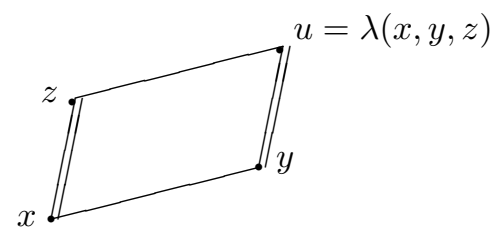

The figure 3 is meant to indicate that the data of $\lambda$ provides a way of closing a whisker $(x, y, z)$ into a parallellogram (one may say that $\lambda$ provides a notion of infinitesimal parallelogram); but note that $\lambda$ is not required to be symmetric in $y$ and $z$, which is why we in the figure use different signatures for the line segments connecting $x$ to $y$ and to $z$, respectively.

Here, a line segment (whether single or double) indicates that the points connected by the line segment are neighbours.

If $x, y, z, u$ are four points in $M$ that come about in the way described, we say that the 4 -tuple form a $\lambda$-parallelogram. The fact that we in the picture did not make the four line segments oriented contains some symmetry assertions, which can be proved by working in a coordinatized situation; namely that the 4 -group $\mathbb{Z}_{2} \times \mathbb{Z}_{2}$ acts on the set of $\lambda$-parallelograms; so for instance $(u, z, y, x)$ is a $\lambda$-parallelogram, equivalently

$$
\lambda(\lambda(x, y, z), z, y)=x .
$$

${ }^{3}$ Note the difference between this figure and the figure (1.2), in which $y$ and $z$ are assumed to be neighbours, and where the parallelogram canonically may be formed. 
On the other hand

$$
\lambda(\lambda(x, y, z), y, z) \sim x
$$

but it will not in general be equal to $x$; its discrepancy from being $x$ is an expression of the torsion of $\lambda$. Even when $y \sim z$ (so $x, y, z$ form a simplex), the left hand side of (2.7) need not be $x$. Rather, we may define the torsion of $\lambda$ to be the law $b$ which to any 2 -simplex $x, y, z$ associates $\lambda(\lambda(x, y, z), y, z)$. Then $b(x, y, z)=x$ for all simplices iff $\lambda$ is symmetric.

There is also a notion of curvature of $\lambda$ : Let $M$ be a manifold equipped with an affine connection $\lambda$. If $x, y, z$ form an infinitesimal 2 -simplex in $M, \lambda(x, y,-)$ takes any neighbour $v$ of $x$ into a neighbour of $y$, and to it, we can then apply $\lambda(y, z,-)$ to obtain a neigbour of $z$. But since $x \sim z$, we could also transport $v$ directly by $\lambda(x, z,-)$. To say that $\lambda$ is flat or curvature-free is to say that we get the same neighbour point of $z$ as the result. An equivalent description of flatness of $\lambda$ is to say that applying $\lambda(x, y,-)$, then $\lambda(y, z,-)$, then $\lambda(z, x,-)$ to any $v \sim x$ will return $v$ as value. In this conception, the curvature $r$ of $\lambda$ is a law, which to any infinitesimal 2 -simplex $x, y, z$ provides a permutation of the set of neighbours of $x$. (In the terminology of [7], $r$ is a group-bundle valued combinatorial 2-form.)

We give two examples of affine connections on the unit sphere $S$.

Example 1. The unit sphere $S$ sits inside Euclidean 3-space, $S \subseteq E$. Since $E$ is in particular an affine space, we may for any three points $x, y, z$ in it form $y-x+z \in E$. For $x, y, z$ in $S$, the point $y-x+z$ will in general be outside $S$; if $x, y, z$ are mutual neighbours, however, $y-x+z$ will be in $S$, cf. Remark at the end of Section 1. What if $x, y, z$ form an infinitesimal 2-whisker? Then we may define $\lambda(x, y, z) \in S$ to be the point, where the half line from the center of $S$ to $y-x+z$ meets $S$. It is easy to see that (2.2) holds. The equation (2.3) requires a little argument in the following spirit: the failure of (2.3) to hold is quadratic in $x-y$, and therfore vanishes because $x-y \in D(3) \subset E=R^{3}$.

This affine connection is evidently symmetric in $y$ and $z$, so is torsion free; it does, however, have curvature. It is the Riemann- or Levi-Civita connection on sphere.

Example 2. (This example does not work on the whole sphere, only away from the two poles.) Given $x, y$ and $z$ with $x \sim z(x \sim y$ is presently not relevant). Since $x$ and $z$ are quite close, we can uniquely describe $z$ in a rectangular coordinate system at $x$ with coordinate axes pointing East and North. Now take $\lambda(x, y, z)$ to be that point near $y$, which in the East-North coordinate system at $y$ has same coordinates as the ones obtained for $z$ in the coordinate system that we considered at $x$.

The description of this affine connection is asymmetric in $y$ and $z$, and it is indeed easy to calculate that it has torsion ([7, Section 2.4). It has no curvature. - One may think of this $\lambda(x, y, z)$ as parallel transporting $z$ from $x$ to $y$; so $x y$ is an active aspect, $z$ is a passive aspect.

Connections constructed in a similar way also occur in materials science: for a crystalline substance one may attach a coordinate system at each point, by using 
the crystalline structure to define directions (call them "East" and "North" and "Up", say). The torsion for a connection $\lambda$ constructed from such coordinate systems is a measure for the imperfection of the crystal lattice (dislocations), see $[8,4$ and the references therein.

\section{Second Order Notions; Midpoint Formation}

The data of an affine connection on a manifold $M$ is a (partially defined) ternary operation $\lambda$. We indicate in this Section how the data of a symmetric (=torsion free) affine connection may be encoded by a binary operation "midpoint formation" $\mu$ on pairs of second order neighbours in $M$.

Let $M_{(2)} \subseteq M \times M$ denote the set of pairs $(x, u)$ of second order neighbours; $M_{(2)}$ is the "second neighbourhood of the diagonal", in analogy with the first neighbourhood $M_{(1)}$ described in Section [1. We have $M_{(1)} \subseteq M_{(2)}$. If $\lambda$ is an affine connection on $M$, then for any 2 -whisker $x, y, z$, we have that $x \sim_{2} \lambda(x, y, z)$.

Recall that for $x \sim_{1} y$ in $M$, we have canonically the affine combination $\frac{1}{2} x+\frac{1}{2} y$, the midpoint.

Definition 3.1. A midpoint formation structure on $M$ is a map $\mu: M_{(2)} \rightarrow M$, extending the canonical midpoint formation for pairs of first order neighbour points.

Thus, $\mu(x, u)$ is defined whenever $x \sim_{2} u$; and $\mu(x, u)=\frac{1}{2} x+\frac{1}{2} u$ whenever $x \sim_{1}$ $u$. It can be proved that such $\mu$ is automatically symmetric, $\mu(x, u)=\mu(u, x)$, and that $\mu(x, u) \sim_{2} x$ and $\sim_{2} u$.

Theorem 3.2. There is a bijective correspondence between midpoint formation structures $\mu$ on $M$, and symmetric (= torsion free) affine connections $\lambda$ on $M$.

Proof sketch. Given $\mu$, and given an infinitesimal 2-whisker $(x, y, z)$. Since $x \sim_{1} y$, we may form the affine combination $2 y-x$ (reflection of $x$ in $y$ ), and it is still a first order neigbour of $x$. Similarly for $2 z-x$. So $(2 y-x) \sim_{2}(2 z-x)$, and so we may form $\mu(2 y-x, 2 z-x)$, and we define

$$
\lambda(x, y, z):=\mu(2 y-x, 2 z-x) .
$$

The relevant picture is here:

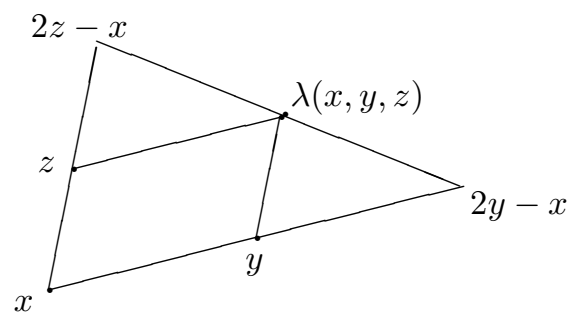


It is symmetric in $y$ and $z$, by the symmetry of $\mu$. Also, if $y=x$, we get

$$
\lambda(x, x, z)=\mu(x, 2 z-x)=\frac{1}{2} x+\frac{1}{2}(2 z-x),
$$

since $x \sim_{1} 2 z-x$ and $\mu$ extends the canonical midpoint formation for first order neighbours. But this equals $z$, by evident equations for affine combinations. This proves the first equation in (2.1), and the second one then follows by symmetry.

The passage from a symmetric affine connection $\lambda$ to midpoint formation $\mu$ is less evident. If $x \sim_{2} u$ and if we have some $y$ which "interpolates" in the sense that $x \sim_{1} y \sim_{1} u$, we may define $\mu(x, u)$ by

$$
\mu(x, u)=\lambda\left(y, \frac{1}{2} x+\frac{1}{2} y, \frac{1}{2} y+\frac{1}{2} u\right)
$$

(make a picture!); one can prove that this does not depend on the choice of the interpolating $y$.

Let us show that one gets the symmetric affine connection $\lambda$ back from the midpoint formation $\mu$ to which it gives rise. Let $\tilde{\lambda}$ be the affine connection constructed from $\mu$, so for a whisker $x, y, z$ at $x$, use $x$ as interpolating point between $2 y-x$ and $2 z-x$; so

$$
\tilde{\lambda}(x, y, z)=\mu(2 y-x, 2 z-x)=\lambda\left(x, \frac{1}{2} x+\frac{1}{2}(2 y-x), \frac{1}{2} x+\frac{1}{2}(2 z-x)\right),
$$

but $\frac{1}{2} x+\frac{1}{2}(2 y-x)=y$ and $\frac{1}{2} x+\frac{1}{2}(2 z-x)=z$, by purely affine calculations; so we get $\lambda(x, y, z)$ back.

Remark. The Theorem may also be seen as a manifestation of a simple algebraic fact: in $R^{n}$, a midpoint formation $\mu$ is given by $\mu(x, y)=\frac{1}{2} x+\frac{1}{2} y+M(x ; y-x)$, where $x \sim_{2} y$ and where $M\left(x ;-\right.$ ) (for each $x$ ) is a function $D_{2}(n) \rightarrow R^{n}$, vanishing on $D_{1}(n)$. By a version of the KL axiom scheme, one sees that each such $M(x ;-)$ extends uniquely to a quadratic $R^{n}$-valued form on $R^{n}$, and there is classically a bijective correspondence between such forms, and symmetric bilinear $R^{n}$-valued forms $\Gamma$ on $R^{n}$; these $\Gamma$ s will serve as Christoffel symbols for the symmetric affine connection corresponding to $\mu$, modulo a factor -8 .

In [5], it is shown how a Riemannian metric geometrically gives rise to a midpoint formation (out of which, in turn, the Levi-Civita affine connection may be constructed, by the process given by the Theorem).

Problem: Since a midpoint formation structure $\mu$ gives rise to an affine connection $\lambda$ by a geometric construction, and an affine connection $\lambda$ gives rise to a curvature $r$, likewise constructed geometrically, one gets by concatenation of these constructions a geometric construction of $r$ out of $\mu$. Is there a more direct geometric way of getting $r$ from $\mu$ ?

\section{References}

1. Breen, L., Messing, W.: Combinatorial differential forms. Advances in Math. 164, 203-282 (2001) 
2. Kock, A.: Synthetic Differential Geometry. London Math. Soc. Lecture Notes Series, vol. 51. Cambridge University Press, Cambridge (1981)*(Second edition, London Math. Soc. Lecture Notes Series, vol. 333. Cambridge University Press, Cambridge (2006))

3. Kock, A.: A combinatorial theory of connections. In: Gray, J. (ed.) Mathematical Applications of Category Theory, Proceedings 1983. AMS Contemporary Math., vol. 30, pp. 132-144 (1984)

4. Kock, A.: Introduction to Synthetic Differential Geometry, and a Synthetic Theory of Dislocations. In: Lawvere, F.W., Schanuel, S. (eds.) Categories in Continuum Physics, Proceedings Buffalo 1982. Springer Lecture Notes, vol. 1174. Springer, Heidelberg (1986)

5. Kock, A.: Geometric construction of the Levi-Civita parallelism. Theory and Applications of Categories 4, 195-207 (1998)

6. Kock, A.: Differential forms as infinitesimal cochains. Journ. Pure Appl. Alg. 154, 257-264 (2000)

7. Kock, A.: Synthetic Geometry of Manifolds. Cambridge Tracts in Mathematics 180 (2009)

8. Noll, W.: Materially uniform simple bodies with inhomogeneities. Arch. Rat. Mech. Anal. 27, 1-32 (1967) 